\title{
On minimal self-joinings in topological dynamics
}

\author{
ANDRÉS DEL JUNCO \\ Department of Mathematics, University of Toronto, Toronto, Canada, M5S 1A1
}

(Received 2 December 1985 and revised 15 June 1986)

\begin{abstract}
If $X$ is a compact metric space and $T$ a homeomorphism of $X$ we say $(X, T)$ has almost minimal power joinings (AMPJ) if there is a dense $G_{\delta} X^{*}$ in $X$ such that for each finite set $k, x \in\left(X^{*}\right)^{k}$ and $1: k \rightarrow \mathbb{Z}-\{0\}$, the orbit closure $\operatorname{cl}\left\{\left(\otimes_{j \in k} T^{1(j)}\right)^{i} x: i \in \mathbb{Z}\right\}$ is a product of off-diagonals (POOD) on $X^{k}$. By an offdiagonal on $X^{k^{\prime}}, k^{\prime} \subset k$ we mean a set of the form $\left(\otimes_{j \in k^{\prime}} T^{m(j)}\right) \Delta, \Delta$ the diagonal in $X^{k^{\prime}}, \mathrm{m}: \boldsymbol{k}^{\prime} \rightarrow \mathbb{Z}$ any function, and by a POOD on $X^{k}$ we mean that $k$ is split into subsets $k^{\prime}$, on each $X^{k^{\prime}}$ we put an off-diagonal and then we take the product of these.

We show that examples of AMPJ exist and that this definition leads to a theory completely analogous to Rudolph's theory of minimal self-joinings in ergodic theory. In particular if $(X, T)$ has AMPJ the automorphism group of $T$ is $\left\{T^{n}\right\}, T$ has only almost 1-1 factors (other than the trivial one) and the automorphism group and factors of $\bigotimes_{i \in k} T, k$ finite or countably infinite, can be very explicitly described. We also discuss $\mathbb{R}$-actions.
\end{abstract}

\section{Introduction}

The aim of this paper is to formulate a definition of minimal self-joinings in topological dynamics which mimics as closely as possible the definition in ergodic theory $([\mathbf{R u}]$ and $[\mathbf{J}, \mathbf{R}])$ and from which a theory precisely parallel to that in $[\mathbf{R u}]$ can be developed.

Let $T$ for the moment denote a measure-preserving automorphism of a probability space $(X, F, \mu)$. For a finite or countably infinite set $k$ and $1: k \rightarrow \mathbb{Z}$ let $T^{1}$ denote the map $\otimes_{i \in k} T^{1(i)}$ of $X^{k}$, that is $\left(T^{1} x\right)(i)=T^{1(i)} x(i)$. A $k$-joining of $T$ is a measure on $X^{k}$ which is invariant under $T^{1}(1(i)=1)$ and all of whose one-dimensional marginals are $\mu$. By an off-diagonal measure on $X^{k^{\prime}}, k^{\prime} \subset k$, we mean a measure of the form $T^{1} \mu_{\Delta}, \mu_{\Delta}$ diagonal measure on $X^{k^{\prime}}$. A product of off-diagonals (POOD) on $X^{k}$ is then a product of off-diagonal measures defined on $X^{k_{1}}, \ldots, X^{k_{r}}$ where $k$ is the disjoint union of $k_{1}, \ldots, k_{r}$. POOD's are evidently $k$-joinings. According to $[\mathbf{J}, \mathbf{R}] T$ has minimal self-joinings (MSJ) if for every finite $k$ each ergodic $k$-joining of $T$ is a POOD. This definition is almost equivalent to the original one in [Ru] (see $[\mathbf{J}, \mathbf{R}]$ for an explanation of the exact relationship). MSJ allows, among other things, a complete description of the centralizer and factor algebras of $T^{\mathbf{l}}$. T is said to have minimal power joinings (MPJ) if for every finite $k$ and $1: k \rightarrow \mathbb{Z}-[0]$ any 
$T^{1}$-invariant and ergodic measure on $X^{k}$ with marginals $\mu$ (that is, an ergodic joining of $\left\{T^{1(i)}: i \in k\right\}$ ) is a POOD. (Note that now not all POOD's are invariant.)

Now let $X$ denote a compact metric space and $T$ a homeomorphism of $X$. Markley [M] and Auslander and Markley $[\mathbf{A}, \mathbf{M}]$ have developed a theory of topological MSJ based on the premise that ergodic joinings correspond to minimal subsets of $X^{k}$. Specifically, they call $(X, T)$ graphic if every non-zero power of $T$ is minimal and every minimal subset of $X \times X$ is a graph $\left\{\left(x, T^{n} x: x \in X\right\}\right.$. Note that $X \times X$, the analogue of product measure, plays no role as it is not minimal. They obtain a number of interesting results, for example if $(Y, S)$ is minimal and not disjoint from $\left(X, T^{m}\right)$ then $\left(X, T^{m}\right)$ is a factor of a proximal extension of $(Y, S)$. This allows one to conclude that $T^{m}$ and $T^{n}$ are disjoint for $m>n>0$. They also obtain a multiple disjointness result for powers of graphic flows ("pairwise disjointness implies multiple disjointness'). However their theory is by it's nature limited to minimal flows. It is, for example, not known whether $T \times T$ and $T \times T \times T$ are non-isomorphic for $T$ graphic, which is certainly true in the measure-theoretic setting (see [Ru]).

Here we work on the premise that ergodic joinings correspond to ergodic (topologically transitive) subsets of $X^{k}$ - in other words we impose a strong restriction on orbit closures in $X^{k}$. It should be noted that if one is only concerned with minimal subsets of $X^{k}$ then it is enough to specify them for $k=2$; one then gets the minimal sets in $X^{k}$ easily. This is not the case here: because $X^{2}$ itself can be ergodic the ergodic subsets of $X^{k}$ cannot be easily derived from knowledge of ergodic subsets of $X^{2}$.

With our definition we are able to recover essentially all of the results of [Ru] with the proviso that results on factors obtain only modulo almost 1-1 homomorphisms. In particular, for example, the automorphism group of $\left(X^{k}, T^{1}\right)$ is generated by the $T^{1}$ and the co-ordinate permutations on $X^{k}$. Moreover if $(Y, S)$ is any factor of $\left(X^{k}, T^{1}\right)$, then $\left(X_{k}, T^{1}\right)$ is a group extension of an almost 1-1 extension of $Y$.

We define off-diagonals and POOD's just as in ergodic theory, that is an offdiagonal on $X^{k}$ is a set of the form $T^{\mathrm{l}} \Delta$ where $\Delta$ is the diagonal in $X^{k}$ and $1: k \rightarrow \mathbb{Z}$, and a POOD is a product of such sets. The most natural definition of minimal power joinings of the flow $(X, T)$ for our purposes would be that for each finite $k$ and $1: k \rightarrow \mathbb{Z}-\{0\}$, each $T^{1}$-orbit closure in $X^{k}$ is a POOD. Unfortunately, we have been unable to find an example satisfying this condition. The natural candidates are the POD flows of $[\mathbf{F}, \mathbf{K}, \mathbf{S}]$ and Chacón's example $[\mathbf{J}],[\mathbf{J}, \mathbf{K}]$ but these all have points $(x, y) \in X^{2}$ whose $T \times T$-orbit closure consists of the orbit itself together with offdiagonals $\left(I \times T^{n}\right) \Delta$. (Moreover it seems .unlikely that any symbolic flow would have this property because such a flow has forward asymptotic points $x$ and $y$ so the future limit set of $(x, y)$ is $\Delta$ and the past limit set would have to be $X \times X)$. However this only happens when $x$ and $y$ both belong to a certain countable exceptional set. This suggests the following definition which is in keeping with the measure-theoretic spirit of throwing out null sets.

We write $\mathscr{O}(x, T)$ for the orbit $\left\{T^{i} x: i \in \mathbb{Z}\right\}$ and $\overline{\mathcal{O}}(x, T)$ for the closure of the orbit. We also write $\overline{\mathcal{O}}_{+\infty}(x, T)$ for the set of limit points of $\left\{T^{n} x: n \in \mathbb{Z}^{+}\right\}$. We will say $(X, T)$ has almost minimal power-joinings (AMPJ) if there is a dense $T$-invariant 
$G_{\delta} X^{*}$ in $X$ such that for each finite set $k, 1: k \rightarrow \mathbb{Z}-\{0\}$ and $x \in\left(X^{*}\right)^{k}$ one has that $\bar{O}\left(x, T^{1}\right)$ is a POOD. (Of course if $T$ is weak-mixing, $T^{1}$ is ergodic so there is a dense $G_{\delta}$ set of points in $X^{k}$ having dense orbit, but our requirement is much stronger).

Some remarks on this definition are in order. If two co-ordinates $i$ and $j$ belong to the same off-diagonal in a POOD $\Delta$ we say they are linked (by $\Delta$ ). Clearly this can only happen when $\mathbf{l}(i)=\mathbf{l}(j)$ and $x(i)=T^{m} x(j)$ for some $m$, in which case we say that $m$ is the link between $i$ and $j$. Thus if $x \in\left(X^{*}\right)^{k}$ and $x(i)$ and $x(j)$ are on different orbits whenever $\mathbf{l}(i)=\mathrm{l}(j)$ then $\overline{\mathcal{O}}\left(x, T^{\mathrm{l}}\right)=X^{k}$. In fact this condition is equivalent to AMPJ. To see this suppose $x \in\left(X^{*}\right)^{k}$ is arbitrary, split $k$ into maximal disjoint subsets $K_{i}$ on each of which $l$ is constant and any two co-ordinates of $x$ are on the same orbit and choose $S \subset k$ intersecting each $K_{i}$ in a singleton. Denoting by $\bar{x}$ the projection of $x$ on $X^{S}$ and by $\bar{l}$ the restriction of $I$ to $S$ we have $\overline{\mathcal{O}}\left(\bar{x}, T^{1}\right)=X^{S}$. It follows that $\bar{O}\left(x, T^{\mathbf{l}}\right)$ is a POOD with off-diagonals sitting precisely on the $X^{K_{i}}$.

AMPJ is sufficient to characterise the centralizer of $T^{1}$ (theorem 3.4), but for our characterisation of factors we seem to need something stronger. We will say $(X, T)$ has strong almost minimal power joinings (SAMPJ) if it has AMPJ with 'good' set $X^{*}$ and if in addition, setting $X^{\prime}=X-X^{*}$ we have for all finite sets $k$ and $k^{\prime}$, $x \in\left(X^{*}\right)^{k}, x^{\prime} \in\left(X^{\prime}\right)^{k^{\prime}}, \mathbf{l}: k \rightarrow \mathbb{Z}-\{0\}$ and $\mathbf{l}^{\prime}: k^{\prime} \rightarrow \mathbb{Z}-\{0\}$ that

$$
\overline{\mathcal{O}}_{+\infty}\left(\left(x, x^{\prime}\right), T^{\mathbf{l}} \times T^{\mathrm{l}^{\prime}}\right)=\overline{\mathcal{O}}_{+\infty}\left(x, T^{\mathrm{l}}\right) \times \overline{\mathcal{O}}_{+\infty}\left(x^{\prime}, T^{\mathbf{l}}\right) .
$$

We hope that this rather ad hoc definition will prove to be a provisional one, conditioned as it is by the results we want to obtain (i.e. the 'Rudolph theory' in the topological setting) and what we are able to show about Chacón's example.

In $\S 2$ we show how SAMPJ of Chacón's example can be quickly deduced from known measure-theoretic properties. In $\S 3$ we explore the consequences of AMPJ along the lines of $[\mathbf{R u}]$ and in $\S 4$ we briefly consider $\mathbb{R}$-actions and mention some open problems.

\section{Chacón's example}

We briefly review the construction of Chacón's example (topological version). The reader is referred to $[\mathbf{J}, \mathbf{K}]$ for proofs. Let $(X, T)$ be the subshift of $\{0,1\}^{\mathbb{Z}}$ defined by the substitution

$$
\theta(0)=0010, \quad \theta(1)=1 .
$$

We set $B_{k}=\theta^{k}(0)$ so $B_{0}=0$ and $B_{k+1}=B_{k} B_{k} 1 B_{k}$. Thus $X$ consists of all two-sided infinite sequences $x$ of 0 's and 1's such that each finite word in $x$ is a word in $B_{k}$ for some $k . X$ is clearly closed and shift-invariant. We now give a very explicit description of $X$.

Sequences in $X$ can be constructed by the following nesting procedure. Fix $\xi \in\{1,2,3,\}^{N}$. Now write down $B_{0}$, nest this as the $\xi(1)$ th $B_{0}$ in $B_{1}$, nest the $B_{1}$ so obtained as the $\xi(2)$ th $B_{1}$ in a $B_{2}$ and so on. This gives rise to an infinite sequence which is well defined up to a shift. If it is a doubly infinite sequence (which is the case unless $\xi(i)=1$ eventually or $\xi(i)=3$ eventually) it evidently belongs to $X$. The unique (up to a shift) left infinite sequence corresponding to $\xi(i)=3$ eventually will 
be denoted by $B_{-\infty}$ and the right infinite one by $B_{\infty} . B_{-\infty} B_{\infty}$ and $B_{-\infty} 1 B_{\infty}$ both belong to $X$ since $B_{k} B_{k}$ and $B_{k} 1 B_{k}$ both occur in $B_{k+1}$.

Proposition 2.1. Every $x \in X$ is up to a shift either a 2-sided infinite nesting of $k$-blocks or $B_{-\infty} B_{\infty}$ or $B_{-\infty} 1 B_{\infty}$.

Proof. See $[\mathbf{J}, \mathbf{K}]$.

The following is an immediate consequence of proposition 2.1 .

Proposition 2.2. $(X, T)$ is minimal and uniquely ergodic.

We denote by $X^{\prime}$ the set of points consisting of the two orbits of the form $B_{-\infty} B_{\infty}$ and $B_{-\infty} 1 B_{\infty}$ and we write $X^{*}$ for $X-X^{\prime}$.

THEOREM 2.3. $(X, T)$ has SAMPJ.

Proof. First, suppose that $x_{1}, x_{2} \in X$ with at most one of $x_{1}$ and $x_{2}$ in $X^{\prime}$ and $m_{1}$, $m_{2} \in \mathbb{Z}-\{0\}$ with $x_{1}$ and $x_{2}$ on different orbits if $m_{1}=m_{2}$. We claim that then $\left(x_{1}, x_{2}\right)$ is $T^{m_{1}} \times T^{m_{2}}$ right-generic for $\mu \times \mu, \mu$ the unique invariant measure on $X$. If $m_{1} \neq m_{2}$ this follows from unique ergodicity of $T^{m_{1}}$ and $T^{m_{2}}$ (which follows from unique ergodicity of $T$ and measure-theoretic weak-mixing of $T$ ) and measure-theoretic disjointness of $T^{m_{1}}$ and $T^{m_{2}}$ (which follows from measure-theoretic MPJ of $T[\mathbf{J}, \mathbf{R}, \mathbf{S}])$. If $m_{1}=m_{2}=1$ this is the main result of $[J, K]$. For general $m_{1}=m_{2}=m$, suppose $\left(x_{1}, x_{2}\right)$ is $T^{m} \times T^{m}$-quasi-generic for a measure $\lambda$, that is there exist $n_{i}>0$ such that

$$
\left(A_{n_{i}}^{m} f\right)\left(x_{1}, x_{2}\right)=\frac{1}{n_{i}} \sum_{j=0}^{n_{i}-1} f \circ\left(T^{m} \times T^{m}\right)^{j}\left(x_{1}, x_{2}\right) \rightarrow \lambda(f)
$$

for each $f \in C\left(X^{2}\right) . \lambda$ is $T^{m} \times T^{m}$-invariant and has marginals $\mu$ (since $T^{m}$ is uniquely ergodic) so by MPJ $\lambda$ is an average of POOD's. In particular $\lambda$ is $T \times$ $T$-invariant. Now

$$
\begin{aligned}
\left(A_{m n_{i}}^{1} f\right)\left(x_{1}, x_{2}\right) & =\frac{1}{m} \sum_{j=0}^{m-1} A_{n_{i}}^{m}\left(f \circ(T \times T)^{j}\right)\left(x_{1}, x_{2}\right) \\
& \rightarrow \frac{1}{m} \sum_{j=0}^{m-1} \lambda\left(f \circ(T \times T)^{j}\right)=\lambda(f) .
\end{aligned}
$$

But

$$
\left(A_{m n_{i}}^{1} f\right)\left(x_{1}, x_{2}\right) \rightarrow(\mu \times \mu)(f)
$$

by $[\mathbf{J}, \mathbf{K}]$ so $\lambda=\mu \times \mu$. Thus $\mu \times \mu$ is the only measure for which $\left(x_{1}, x_{2}\right)$ is $T^{m} \times$ $T^{m}$-quasi-generic whence $\left(x_{1}, x_{2}\right)$ is $T^{m} \times T^{m}$-generic for $\mu \times \mu$.

Now suppose $x=\left(x_{1}, \ldots, x_{k}\right) \in X^{k}$ with at most one co-ordinate in $X^{\prime}$ and $\mathbf{m}: k \rightarrow \mathbb{Z}-\{0\}$ with $\mathbf{m}(i) \neq \mathbf{m}(j)$ whenever $x_{i}$ and $x_{j}$ are on the same orbit. Suppose $x$ is $T^{\mathrm{m}}$-quasi-generic for $\lambda$. By our previous observation each 2-dimensional marginal of $\lambda$ is $\mu \times \mu$. By MSJ $\lambda$ is an average $\sum a_{i} \lambda_{i}$ of POOD's. If $\vec{\lambda}_{i}$ denotes the marginal of $\lambda_{i}$ on some pair of co-ordinates then $\sum a_{i} \bar{\lambda}_{1}=\mu \times \mu$ so all $\bar{\lambda}_{i}$ are $\mu \times \mu$ by ergodicity of $\mu \times \mu$. Thus any 2-dimensional marginal of $\lambda_{i}$ is $\mu \times \mu$ so each $\lambda_{i}$ is $\mu^{k}$, that is $\lambda=\mu^{k}$. We have shown $\mu^{k}$ is the only measure for which $x$ is quasi-generic, so in 
fact $x$ is generic for $\mu^{k}$. It follows that $X^{k}=\operatorname{supp} \mu^{k} \subset \overline{\mathcal{O}}_{+\infty}\left(x, T^{\mathrm{m}}\right)$ so $\overline{\mathcal{O}}_{+\infty}\left(x, T^{\mathrm{m}}\right)=$ $X^{k}$.

To complete the proof of SAMPJ suppose now that $x \in\left(X^{*}\right)^{k}, x^{\prime} \in\left(X^{\prime}\right)^{k^{\prime}}, 1: k \cup$ $k^{\prime} \rightarrow \mathbb{Z}-0$. Divide $k$ into maximal subsets $K_{j}$ on each of which $x_{i}$ are all on the same orbit and $n_{i}$ is constant. Choose a subset $S$ of $k \cup k^{\prime}$ intersecting each $K_{j}$ and $k^{\prime}$ in a singleton. By what we have shown

$$
\overline{\mathcal{O}}_{+\infty}\left(\bar{x}, \otimes_{i \in S} T^{1(i)}\right)=X^{s}
$$

where $\bar{x}$ denotes the projection of $\left(x, x^{\prime}\right)$ on $X^{S}$. It follows that $\bar{O}\left(x, \otimes_{i \in k} T^{1(i)}\right)$ is a POOD, with off-diagonals sitting precisely on the $X^{K_{j}}$, establishing AMPJ. Moreover since any two $x(i), i \in k^{\prime}$, are, up to a shift, forward asymptotic, similar reasoning shows that $\overline{\mathcal{O}}_{+\infty}\left(\left(x, x^{\prime}\right), T^{l}\right)$ splits as the product of its projections on $X^{k}$ and $X^{k^{\prime}}$.

It should certainly be possible to prove theorem 2.3 directly. We were in fact able to go fairly far in this direction, in particular to characterize the orbit closures when 1 is constant, which already is a lot of information. In particular, it gives full information about the centralizer and factors of $T^{1}$ and shows, for example, that $T \times T \times T$ and $T \times T$ are not isomorphic, answering a question raised by Markley [M]. However the arguments became involved and the full generality eluded us so we opted to lean on $[\mathbf{J}, \mathbf{K}]$ and $[\mathbf{J}, \mathbf{R}, \mathbf{S}]$.

\section{Consequences of AMPJ}

Let us say that $(X, T)$ has almost minimal self-joinings (AMSJ) if it satisfies the definition of AMPJ with $\mathbf{l}=1$. Translation by 1 on $\mathbb{Z} / m \mathbb{Z}$ evidently has AMSJ. More generally if $(X, T)$ has a single orbit which is a dense $G_{\delta}$ (in this case we say $(X, T)$ is almost transitive) then $(X, T)$ has AMSJ. This trivial case is the only one which is not weakly mixing.

Proposition 3.1. If $(X, T)$ has AMSJ then it is either almost transitive or weak-mixing. Proof. Suppose that $(X, T)$ is not almost transitive. Since $(X, T)$ is ergodic (this is built into the definition of AMSJ) it is easy to see that it can have no isolated points. It follows that each off-diagonal $\Delta_{n}=\left\{\left(x, T^{n} x\right): x \in X\right\}$ is a closed nowhere dense set in $X^{2} . X^{*} \times X^{*}$ is a dense $G_{\delta}$ in $X \times X$ so $X^{*} \times X^{*}-\cup_{n} \Delta_{n}$ is also a dense $G_{\delta}$. But any point in this set has a dense $T \times T$-orbit, so $(X, T)$ is weak-mixing.

Henceforth we will work only with weak-mixing AMSJ.

If $\phi:(X, T) \rightarrow(Y, S)$ is a factor map we say it is almost 1-1 if $\left(x: \phi^{-1}\{\phi(x)\}=\{x\}\right)$ is dense in $X$. This set is always a $G_{\delta}$ (its complement is the $F_{\sigma}$ consisting of points $x$ such that for some $n$ the diameter of $\phi^{-1}\{\phi(x)\}$ is greater than or equal to $\left.1 / n\right)$ so if $\phi$ is almost $1-1$ it is a dense $G_{\delta}$.

Proposition 3.2. If $\phi:(X, T) \rightarrow(Y, S)$ is almost 1-1 and either $(X, T)$ or $(Y, S)$ has AMSJ or AMPJ then so does the other.

Proof. Let $X^{\prime}=\left(x: \phi^{-1}\{\phi(x)\}=\{x\}\right)$, which is a dense $G_{\delta}$ in $X$, and $Y^{\prime}=\phi\left(X^{\prime}\right) . Y^{\prime}$ is also a dense $G_{\delta}$ since $Y-Y^{\prime}=\phi\left(X-X^{\prime}\right)$ is an $F_{\sigma}$. An easy compactness argument 
shows that $\phi^{-1}: Y^{\prime} \rightarrow X^{\prime}$ is a homeomorphism. If $(Y, S)$ has AMSJ or AMPJ with 'good set' $Y^{*}, Y^{*} \cap Y^{\prime}$ is a dense $G_{\delta}$ in $Y$, hence also in the relative topology on $Y^{\prime}$. Thus if we set $X^{*}=\phi^{-1}\left(Y^{*} \cap Y^{\prime}\right), X^{*}$ is a dense $G_{\delta}$ in $X^{\prime}$ and hence in $X$. It is then easy to check that $X^{*}$ provides a good set for $(X, T)$. Similarly if $(X, T)$ has AMSJ with good set $X^{*}, \phi\left(X^{*} \cap X^{\prime}\right)$ is a good set for $(Y, S)$.

The rest of the results in this section are simply a faithful translation of the results of [Ru]. The proofs of the key results are, however, quite different. In [Ru] once these key results are obtained the rest is computational. This is the case here also with formally identical computations which we shall leave as an exercise for the interested reader. We shall follow the notation of [Ru] quite closely.

For the rest of this section we fix a weak mixing $(X, T)$ with AMPJ. If $K$ and $K^{\prime}$ are finite or countably infinite sets and $\alpha: k \rightarrow k^{\prime}$ is $1-1$ and onto we denote by $S_{\alpha}$ the homeomorphism $x \rightarrow x \circ \alpha^{-1}$ of $X^{k}$ to $X^{k^{\prime}}$. For $\mathbf{m}: k \rightarrow \mathbb{Z}$ we write $U(\alpha, \mathbf{m})$ for $S_{\alpha} T^{\mathrm{m}}$. Note that $S_{\alpha} T^{\mathrm{m}}=T^{\mathrm{m} \circ \alpha^{-1}} S_{\alpha}$.

The maps we shall analyze in the sequel are the $U(\pi, 1)$ for $\pi: k \rightarrow k$ a permutation and $\mathbf{l}: k \rightarrow \mathbb{Z}$. We impose the conditions that $\pi$ have finite cycles and the sum of 1 over each cycle be non-zero. The first condition is equivalent to the requirement that $S_{\pi}$ generate a group whose closure in the uniform topology is compact. We call such a $\pi$ compact. The second condition amounts to requiring that $U(\pi, 1)$ be weak-mixing. Indeed if $k$ is finite and $\pi$ has a single cycle then $U(\pi, 1)^{|k|}=T^{\Sigma 1}$ where $\Sigma=\sum_{i \in k} \mathbf{l}(i)$. Thus $U(\pi, 1)$ is weak-mixing if and only if $T^{\Sigma 1}$ is, which is equivalent to $\Sigma \neq 0$, since $T$ itself is weak-mixing. In general a $U(\pi, 1)$ splits as a product of $U\left(\pi_{i}, \mathbf{l}_{i}\right)$ 's corresponding to the cycles of $\pi$. Note also that weak-mixing and ergodicity of $U(\pi, 1)$ are equivalent.

LEMMA 3.3. If $\pi: k \rightarrow k$ is compact, $U(\pi, \mathbf{l})$ is ergodic and $x \in\left(X^{*}\right)^{k}$ has no two co-ordinates on the same T-orbit then $\bar{O}(x, U(\pi, 1))=X^{k}$.

Proof. Let $k_{i} \uparrow k$ be finite unions of orbits of $\pi$ so that $U(\pi, 1)=U_{i} \times \bar{U}_{i}$ on $X^{k_{i}} \times X^{k-k_{i}}$. Let $\sigma_{i}: X^{k} \rightarrow X^{k_{i}}$ denote the projection, $E=\bar{O}(x, U(\pi, 1))$ and $E_{i}=\sigma_{i}(E)=$ $\overline{\mathcal{O}}\left(\sigma_{i}(x), U_{i}\right)$. Choosing $m$ such that $U_{i}^{m}=T^{\mathbf{N}}$ for some $\mathbf{N}: k_{i} \rightarrow \mathbb{Z}-\{0\}$ we have $E_{i} \supset \overline{\mathcal{O}}\left(x_{i}, T^{\mathrm{N}}\right)$. But $\overline{\mathcal{O}}\left(x_{i}, T^{\mathrm{N}}\right)=X^{k_{i}}$ since $x_{i}$ has no two co-ordinates on the same orbit. Thus $\pi_{i}(E)=X^{k_{i}}$ for all $i$ and, since $E$ is closed, it follows that $E=X^{k}$.

We shall work with a weak notion of isomorphism which is natural in the context of our definition of AMPJ. We'll say $(X, T)$ and $(Y, S)$ are weakly isomorphic via the map $\phi$ if there are invariant dense $G_{\delta}$ subsets $E^{*}$ and $F^{*}$ in $X$ and $Y$ and $\phi$ is a homeomorphism from $E^{*}$ to $F^{*}$ such that $\phi T x=S \phi x$ for $x \in E^{*}$. For example, if $(X, T)$ and $(Y, S)$ have a common almost 1-1 factor or a common almost 1-1 extension they are weakly isomorphic by the observations in the proof of proposition 3.2. Note that ergodicity and weak-mixing are invariants of weak isomorphism. One consequence of the following theorem is that $U(\pi, 1)$ and $U\left(\pi^{\prime}, l^{\prime}\right)$ are weakly isomorphic if and only if they are isomorphic.

THEOREM 3.4. Suppose $U(\pi, 1)$ and $U\left(\pi^{\prime}, \mathbf{l}^{\prime}\right)$ are weak-mixing on $X^{k}$ and $X^{k^{\prime}}$ with $\pi$ and $\pi^{\prime}$ compact. If $U(\pi, 1)$ and $U\left(\pi^{\prime}, \mathrm{l}^{\prime}\right)$ are weakly isomorphic via $\phi$ then there is 
an $\alpha: k \rightarrow k^{\prime} 1-1$ and onto and $\mathbf{m}: k \rightarrow \mathbb{Z}$ such that $\phi$ and $U(\alpha, \mathrm{m})$ agree on the domain of $\phi$. In particular $U(\alpha, \mathbf{m})$ is a continuous isomorphism between $U(\pi, \mathbf{l})$ and $U\left(\pi^{\prime}, \mathbf{l}^{\prime}\right)$.

Proof. Suppose $\phi: G \rightarrow G^{\prime}$ homeomorphically where $G$ and $G^{\prime}$ are invariant dense $G_{\delta}$ subsets of $X^{k}$ and $X^{k^{\prime}}$. Let $D \subset X^{k}\left(D^{\prime} \subset X^{k^{\prime}}\right)$ denote those points having no pair of co-ordinates on the same $T$-orbit. $D$ and $D^{\prime}$ are dense $G_{\delta}$ 's in $X^{k}$ and $X^{k^{\prime}}$. Thus $\left(X^{*}\right)^{k^{\prime}} \cap D^{\prime} \cap G^{\prime}$ is a relative dense $G_{\delta}$ in $G^{\prime}$ so its inverse image under $\varphi$ is a relative dense $G_{\delta}$ in $G$, hence also a dense $G_{\delta}$ in $X^{k}$. It follows that we may choose $\bar{x} \in\left(X^{*}\right)^{k} \cap D \cap G$ so that $\phi(\bar{x}) \in\left(X^{*}\right)^{k^{\prime}} \cap D^{\prime} \cap G^{\prime}$.

We set $U=U(\pi, \mathbf{l}), U^{\prime}=U\left(\pi^{\prime}, \mathbf{l}^{\prime}\right)$ and $E=\overline{\mathcal{O}}\left((\bar{x}, \phi(\bar{x})), U \times U^{\prime}\right)$. Note that $E \cap$ $G \times X^{k^{\prime}}=\{(x, \phi(x)): x \in G\}$. This follows from lemma 3.3 and the fact that $\phi$ is an isomorphism between $\left.U\right|_{G}$ and $\left.U^{\prime}\right|_{G^{\prime}}$. Similarly, $E \cap X^{k} \times G^{\prime}=\{(x, \phi(x)): x \in G\}$. It follows that $\left(E, U \times U^{\prime}\right)$ is weakly isomorphic to $\left(X^{k}, U\right)$ and hence weakly mixing.

Now let $k_{i} \uparrow k$ and $k_{i}^{\prime} \uparrow k^{\prime}$ be finite unions of cycles of $\pi$ and $\pi^{\prime}$ respectively so that we have $U=U_{i} \times \bar{U}_{i}$ on $X^{k_{i}} \times X^{k-k_{i}}$ and $U^{\prime}=U_{i}^{\prime} \times \bar{U}_{i}^{\prime}$ on $X^{k_{i}^{\prime}} \times X^{k^{\prime}-k_{i}^{\prime}}$. Let $\sigma_{i}: X^{k} \times X^{k^{\prime}} \rightarrow X^{k_{i}} \times X^{k_{i}^{\prime}}$ denote the projection $\left(x_{i}, x_{i}^{\prime}\right)=\sigma_{i}(\bar{x}, \varphi(\bar{x}))$ and $E_{i}=\bar{O}\left(\left(x_{i}, x_{i}^{\prime}\right), U_{i} \times U_{i}^{\prime}\right)=\sigma_{i}(E)$. Choosing $n$ such that $\left(\left(U_{i} \times U_{i}\right)^{n}=T^{\mathbf{M}}\right.$, $\mathbf{M}: k_{i} \cup k_{i}^{\prime} \rightarrow \mathbb{Z}-\{0\}$, we have $E_{i} \supset F_{i}=\overline{\mathcal{O}}\left(\left(x_{i}, x_{i}^{\prime}\right), T^{\mathbf{M}}\right)$, which is a POOD. Since $\bigcup_{l=0}^{n-1}\left(U_{i} \times U_{i}^{\prime}\right)^{l} F_{i}=E_{i}$, one of the $\left(U_{i} \times U_{i}^{\prime}\right)^{l} F_{i}$, and hence $F_{i}$ itself, must have interior in $E_{i}$. But $\left(E_{i}, U_{i} \times U_{i}^{\prime}\right)$ is a factor of $\left(E, U \times U^{\prime}\right)$ so it is weak-mixing, whence $\left(E_{i}, T^{\mathbf{M}}\right)$ is also weak-mixing and hence ergodic. Thus the $T^{\mathbf{M}}$-invariant subset $F_{i}$, which has interior, must be all of $E_{i}$.

We have shown that $E_{i}$ is a POOD and it must have full projections on $X^{k_{i}}$ and $X^{k_{i}^{\prime}}$ since no two co-ordinates of $\bar{x}$ or of $\phi(\bar{x})$ are on the same orbit. Thus $t \mathrm{l}$ POOD $E_{i}$ links co-ordinate $j \in k_{i}$ with $\alpha_{i}(j) \in k_{i}^{\prime}$ where $\alpha_{i}$ is a bijection from a subset of $k_{i}$ to a subset of $k_{i}^{\prime}$. Evidently $\alpha_{i+1}$ is consistent with $\alpha_{i}$ so the $\alpha_{i}$ define a bijection $\alpha$ from a subset of $k$ to a subset of $k^{\prime}$. Since $E$ is closed, $E=\bigcap_{i} \pi_{i}^{-1} \pi_{i}(E)$, whence $E$ is a POOD linking $j \in k$ with $\alpha(j)$. We claim that in fact $\alpha$ maps all of $k$ to all of $k^{\prime}$. Indeed if some co-ordinate $i \in k$ were left unlinked by $E$, choose $x, \tilde{x} \in G$ such that $x(i) \neq \tilde{x}(i)$ but $x(j)=\tilde{x}(j)$ for $j \in k-\{i\}$ (this is certainly possible when $G$ is a dense $\left.G_{\delta}\right)$. Since $E \cap G \times X^{k^{\prime}}=\{(x, \phi(x)): x \in G\}$ we have $(x, \phi(x)) \in E$. But we also have $(\tilde{x}, \phi(x)) \in E$, by our choice of $i$. We conclude $\phi(x)=\phi(\tilde{x})$, contradicting injectivity of $\phi$. Similarly no co-ordinate in $k^{\prime}$ could be unlinked.

Thus there is an $\mathbf{m}: k \rightarrow \mathbb{Z}$ such that $E=\left\{(x, U(\alpha, \mathbf{m}) x): x \in X^{k}\right\}$. Since $E \cap G \times X^{k}=\{(x, \phi(x)): x \in G\}, U(\alpha, \mathbf{m})$ and $\phi$ agree on $G$.

Remark. It is easy to show, just as in [Ru], that there is a $U(\alpha, \mathbf{m})$ conjugating $U(\pi, 1)$ and $U\left(\pi^{\prime}, l^{\prime}\right)$ if and only if $\alpha$ conjugates $\pi$ and $\pi^{\prime}$ and $\mathbf{l}$ and $l^{\prime} \circ \alpha$ have the same sum over each cycle of $\pi$. It follows, for example, that $T \times T$ and $T \times T \times T$ are not isomorphic, answering, at least in the case where $T$ has AMPJ, a question raised in $[\mathbf{M}]$.

LEMMA 3.5. (a) If $U(\pi, 1)$ is ergodic on $X^{k}$ with $\pi$ compact and $x \in\left(X^{*}\right)^{k}$ then $\overline{\mathcal{O}}\left((x, U(\pi, 1))\right.$ is a union of POOD's. If on some $\pi$-invariant subset $k^{\prime}$ of $k$, all co-ordinates of $x$ are on different T-orbits then each of these POOD's projects onto all of $X^{k^{\prime}}$. 
(b) Assume $(X, T)$ has SAMPJ and $U(\pi, 1)$ is ergodic on $X^{k}$ with $\pi$ compact. Suppose $x \in X^{k}$, set $k^{\prime}=\left\{i \in k: x(i) \in X^{\prime}\right\}, k^{*}=k-k^{\prime}$ and suppose that on some $\vec{k} \subset k^{*}$ no two co-ordinates of $x$ are on the same orbit. Then $\overline{\mathcal{O}}_{+\infty}((x, U(\pi, 1)) \supset \Delta \times \mathrm{F}$ where $\Delta$ is a POOD on $X^{k^{*}}$ whose projection on $X^{\bar{k}}$ is all of $X^{\bar{k}}$ and $F$ is a non-empty subset of $X^{k^{\prime}}$.

Proof. (a) Let $k_{i} \uparrow k$ be finite unions of cycles of $\pi$. Let $U_{i}$ denote $U\left(\left.\pi\right|_{k_{i}},\left.1\right|_{k_{i}}\right), \sigma_{i}$ the projection onto $X^{k_{i}}$ and $x_{i}=\sigma_{i}(x)$. Let $N_{i} \in \mathbb{Z}^{+}$be such that if $M_{i}=N_{1} N_{2} \cdots N_{i}$ then $U_{i}^{M_{i}}=T^{\mathbf{m}_{i}}, \mathbf{m}_{i}: k_{i} \rightarrow \mathbb{Z}-\{0\}$. Now

$$
\overline{\mathcal{O}}\left(x_{1}, U_{1}\right)=\bigcup_{n=0}^{N_{1}-1} U_{1}^{n} \overline{\mathcal{O}}\left(x_{1}, T^{\mathrm{m}_{1}}\right) \text {. }
$$

We set $X_{n}=U_{1}^{n} \overline{\mathcal{O}}\left(x_{1}, T^{\mathrm{m}_{1}}\right)$ for $0 \leq n<N_{1}$ and observe that the $X_{n}$ are POOD's.

Similarly

$$
\begin{aligned}
\overline{\mathcal{O}}\left(x_{2}, U_{2}\right) & =\bigcup_{n=0}^{M_{2}-1} U_{2}^{n} \overline{\mathcal{O}}\left(x_{2}, T^{\mathrm{m}_{2}}\right) \\
& =\bigcup_{n_{2}=0}^{N_{2}-1} \bigcup_{n_{1}=0}^{N_{1}-1} U_{2}^{n N_{1}} U_{2}^{n_{1}} \overline{\mathcal{O}}\left(x_{i}, T^{\mathbf{m}_{2}}\right) .
\end{aligned}
$$

We set $X_{n_{1}, n_{2}}=U_{2}^{n_{2} N_{1}} U_{2}^{n_{1}} \bar{O}\left(x_{2}, T^{m_{2}}\right)$ and again the $X_{n_{1}, n_{2}}$ are all POOD's. Moreover since $\left.\mathbf{m}_{2}\right|_{k_{1}}=N_{2} \mathbf{m}_{1}, \bar{O}\left(x_{2},\left.T^{\mathbf{m}_{2}}\right|_{k_{1}}\right)=\overline{\mathcal{O}}\left(x_{1}, T^{\mathbf{m}_{1}}\right)$ (any links in these two POOD's must be the same), that is, $\sigma_{1} X_{n_{1}, n_{2}}=X_{n_{1}}$.

Continuing in this way we see that

$$
\sigma_{i} \overline{\mathcal{O}}(x, U(\pi, 1))=\overline{\mathcal{O}}\left(x_{i}, U_{i}\right)=\bigcup_{0 \leq n_{j}<N_{j}} X_{n_{1}, \ldots, n_{i}}
$$

where each $X_{n_{1}, \ldots, n_{i}}$ is a POOD and $X_{n_{1}, \ldots, n_{i}}$ projects onto $X_{n_{1}, \ldots, n_{i-1}}$. It follows that

$$
\overline{\mathcal{O}}(x, U(\pi, \mathbf{l}))=\bigcup_{0 \leq n_{j} \leq N_{j}} X_{n_{1}, n_{2}, \ldots},
$$

where $X_{n_{1}, n_{2}, \ldots}$ is the unique POOD whose projection on each $X^{k_{i}}$ is $X_{n_{1}, \ldots, n_{i}}$.

Finally if $k^{\prime}$ is as described then evidently the projection of $X_{n_{1}, \ldots, n_{i}}$ on $X^{k^{\prime} \cap k_{i}}$ is $X^{k^{\prime} \cap k_{i}}$ for each $i$, whence the projection of $X_{n_{1}, n_{2}, \ldots}$ on $X^{k^{\prime}}$ is $X^{k^{\prime}}$. This completes the proof of (a).

(b) Let $k_{i}, U_{i}, N_{i}, M_{i}$ and $\mathbf{m}_{i}$ be as in the proof of part (a). Let $\sigma_{i}, \sigma_{i}^{\prime}, \sigma_{i}^{*}$ and $\bar{\sigma}_{i}$ denote the projections on $X^{k_{i}}, X^{k_{i} \cap k^{\prime}}, X^{k_{i} \cap k^{*}}$ and $X^{k_{i} \cap \bar{k}}$ respectively. As in (a) we have

$$
\begin{aligned}
\sigma_{i} \overline{\mathcal{O}}_{+\infty}(x, U(\pi, \mathbf{l})) & \supset \sigma_{i} \overline{\mathcal{O}}_{+\infty}\left(x, U(\pi, \mathbf{l})^{M_{i}}\right) \\
& =\overline{\mathcal{O}}_{+\infty}\left(\sigma_{i}(x), T^{\mathbf{m}_{i}}\right),
\end{aligned}
$$

and by SAMPJ this last orbit closure is of the form $\Delta_{i} \times F_{i}$, where $\Delta_{i}$ is a POOD on $X^{k^{*} \cap k_{i}}$ and $F_{i} \subset X^{k_{i} \cap k^{\prime}}$ is the orbit closure of $\sigma_{i}^{\prime}(x)$ under $T^{m_{i}^{\prime}}, \mathbf{m}_{i}^{\prime}$ the restriction of $\mathrm{m}_{i}$ to $k^{\prime} \cap k_{i}$. Moreover $\Delta_{i}$ has no links within $\bar{k} \cap k_{i}$. As in the proof of (a) there is a POOD $\Delta$ on $X^{k^{*}}$, with no links within $\bar{k}$, such that $\sigma_{i}^{*} \Delta=\Delta_{i}$.

Turning to the $F_{i}$ we have $\sigma_{i}\left(F_{i+1}\right) \subset F_{i}$, since $\sigma_{i}\left(F_{i+1}\right)$ is $\bar{O}\left(\sigma_{i}(x), T^{N_{i}+m_{i}}\right)$. Thus if we set $\bar{F}_{i}=\bigcap_{j} \sigma_{i}^{\prime}\left(F_{j}\right)$ we have $\bar{F}_{i} \subset F_{i}$ and $\bar{F}_{i}$ is non-empty by compactness. Since $\sigma_{i+1}^{\prime}\left(F_{j}\right) \searrow \bar{F}_{i+1}$ an easy compactness argument shows that $\sigma_{i}^{\prime}\left(F_{j}\right)=$ $\sigma_{i}^{\prime} \sigma_{i+1}^{\prime}\left(F_{j}\right) \searrow \sigma_{i}^{\prime}\left(\bar{F}_{i+1}\right)$, that is $\bar{F}_{i}=\sigma_{i}^{\prime}\left(\bar{F}_{i+1}\right)$. Another easy compactness argument 
then shows that there is a closed set $F \subset^{\prime} X^{k^{\prime}}$ such that $\sigma_{i}^{\prime}(F)=\bar{F}_{i}$. (This is the analogue of the existence of a measure with prescribed finite dimensional marginals). We have for each $i$

$$
\begin{aligned}
\sigma_{i}(\Delta \times F) & =\Delta_{i} \times \bar{F}_{i} \subset \Delta_{i} \times F_{i} \\
& \subset \sigma_{i} \overline{\mathcal{O}}(x, U(\pi, 1)),
\end{aligned}
$$

so we conclude $\Delta \times F \subset \overline{\mathcal{O}}(x, U(\pi, 1))$.

Suppose $(Z, U)$ is a flow and $G$ is a subgroup of the group of homeomorphisms of $Z$ which is compact in the topology of uniform convergence and $U G U^{-1}=G$. Then the $G$-orbit relation in $Z \times Z$

$$
R_{G}=\{(z, g z): z \in Z, g \in G\}
$$

is a closed, $U$-invariant equivalence relation. Thus we can form the compact metric space $Z / G=Z / R_{G}$ of $G$-orbits, $U$ acts on $Z / G$ and the projection $(Z, U) \rightarrow$ $(Z / G, U)$ is a homomorphism.

We now want to analyze homomorphisms $\phi:\left(X^{k}, U(\pi, 1)\right) \rightarrow(Y, S)$. We say $\phi$ is essential if it depends on all the co-ordinates in $X^{k}$, that is it does not factor through the projection $X^{k} \rightarrow X^{k^{\prime}}$ for any $k^{\prime} \subset k$. We denote by $G(k)$ the group consisting of all $U(\pi, 1)$ on $X^{k}(\pi$ not necessarily compact).

Theorem 3.6. Suppose $(X, T)$ has SAMPJ. Suppose $U=U(\pi, \mathrm{l})$ on $X^{k}$ is ergodic, $\pi$ compact and $\phi:\left(X^{k}, U\right) \rightarrow(Y, S)$ is an essential factor map. Let $G=G(\phi)=$ $\{V \in G(k): \phi V=\phi\}$. Then $G$ is compact $U G U^{-1}=G$ and $\phi$ is the composition of the projection $X^{k} \rightarrow X^{k} / G$ and an almost 1-1 homomorphism from $\left(X^{k} / G, U\right) \rightarrow(Y, S)$. In particular $\left(X^{k} / G, U\right)$ and $(Y, S)$ are weakly isomorphic.

Remark. It follows that $\left(X, T^{n}\right)$ has only almost 1-1 factors for $n \neq 0$. Of course Chacón's example is actually POD and hence has no non-trivial factors at all.

For $k=1$, then, theorem 3.6 does not provide the strongest possible information about factors, because of the presence of an unspecified almost 1-1 homomorphism in the description of $\phi$. Note, however, that for $k>1$, these arise unavoidably. $T \times T$ always has almost 1-1 factors: for example consider the factor map which identifies $(x, x)$ with $(x, T x)$ for each $x \in X$, or the factor map which identifies the diagonal in $X \times X$ to a single point.

Proof. We identify $\left(X^{k}\right)^{2}$ with $X^{k} \times X^{k^{\prime}}=X^{k \cup k^{\prime}}$ where $k^{\prime}$ is a disjoint copy of $k$. Let

$$
R=\left\{(x, y) \in X^{k} \times X^{k^{\prime}}: \phi(x)=\phi(y)\right\}
$$

be the closed $U \times U$-invariant equivalence relation determined by $\phi$. Write $x \sim y$ if $(x, y) \in R$. We evidently have $R \supset R_{G}$.

Let $E$ denote the intersection of $\left(X^{*}\right)^{k}$ with the set of points in $X^{k}$ having no two co-ordinates on the same $T$-orbit and note that $E$ is a dense $G_{\delta}$ in $X^{k}$. Suppose that $\left(x_{1}, x_{2}\right) \in R$ with $x_{1} \in E$. We claim that then $x_{2}$ must be in $\left(X^{*}\right)^{k^{\prime}}$. To see this, suppose that $x_{2} \notin\left(X^{*}\right)^{k^{\prime}}$, so $x(i) \in X^{\prime}$ for $i \in k^{\prime \prime} \subset k^{\prime}$. Set $k^{*}=k^{\prime}-k^{\prime \prime}$. By lemma 3.5 (b), $\bar{O}_{+\infty}\left(\left(x_{1}, x_{2}\right), U \times U\right) \subset R$ contains a set $\Delta \times F, \Delta$ a POOD on $X^{k \cup k^{*}}$ with no links within $k$ (since we assumed $x_{1} \in E$ ) and $F$ a non-empty subset of $X^{k^{\prime \prime}}$. First we want to show that $\Delta$ leaves no co-ordinate in $k$ unlinked. Indeed if $\Delta$ left $i \in k$ 
unlinked then for any $x \in X^{k}$ there is a $y \in X^{k^{\prime}}$ with $(x, y) \in \Delta \times F$, and if $x^{\prime} \in X^{k}$ agrees with $x$ except at $i$ we also have $\left(x^{\prime}, y\right) \in \Delta \times F$. Since $\Delta \times F \subset R$ we have $\phi(x)=\phi(y)=\phi\left(x^{\prime}\right)$ so $\phi$ does not depend on $x(i)$, contradicting essentiality. Thus $\Delta$ links each co-ordinate in $k$ to at least one co-ordinate in $k^{*}$.

This means there is a (potentially) one-to-many map $\alpha$ from $k$ to $k^{\prime}$, whose range is contained in $k^{*}$ such that $\Delta$ links $i \in k$ with all co-ordinates in $\alpha(i)$. Moreover $\alpha(i) \cap \alpha(j)=\varnothing$ for $i \neq j$ (if $i$ and $j$ are linked to a common co-ordinate in $k^{*}$ then they are linked to each other). Recall now that $k^{\prime}$ is a copy of $k$ so it makes sense to iterate $\alpha$. We leave it as an exercise to show that $k^{\prime \prime}, \alpha\left(k^{\prime \prime}\right), \alpha^{2}\left(k^{\prime \prime}\right), \ldots$ are pairwise disjoint (it follows from the fact that $k^{\prime \prime} \cap$ range $\alpha=\varnothing$ ). In particular if $S$ is any finite subset of $k, \alpha^{n}\left(k^{\prime \prime}\right) \cap S=\varnothing$ for sufficiently large $n$. Now suppose that $y_{0}$ and $y_{0}^{\prime}$ belong to $X^{k}$ and agree except on $k^{\prime \prime}$. Then because of the form of $\Delta \times F$ we can find $y_{1}$ and $y_{1}^{\prime}$ such that $\left(y_{0}, y_{1}\right)$ and $\left(y_{0}^{\prime}, y_{1}^{\prime}\right)$ belong to $\Delta \times F$ and $y_{1}$ and $y_{1}^{\prime}$ agree except on $\alpha\left(k^{\prime \prime}\right)$. (To see this observe that the requirement $\left(y_{0}, y_{1}\right) \in \Delta \times F$ specifies the co-ordinates of $y_{1}$ on $\alpha(k)$ and the remaining co-ordinates of $y_{1}$ may be filled in with an arbitrary choice from $F \times X^{k^{*}-\alpha(k)}$. Use the same choice for $y_{1}$ and $y_{1}^{\prime}$ ). Continuing in this way we obtain $y_{0}, y_{1}, y_{2}, \ldots$ and $y_{0}^{\prime}, y_{1}^{\prime}, y_{2}^{\prime}, \ldots$ such that $y_{n}$ and $y_{n}^{\prime}$ agree except on $\alpha^{n}\left(k^{\prime \prime}\right)$ and $\left(y_{n}, y_{n+1}\right)$ and $\left(y_{n}^{\prime}, y_{n+1}^{\prime}\right)$ are in $\Delta \times F$. Since $\Delta \times F \subset R$ we conclude $\phi\left(y_{0}\right)=\phi\left(y_{n}\right)$ and $\phi\left(y_{0}^{\prime}\right)=\phi\left(y_{n}^{\prime}\right)$. Passing to a subsequence we may assume $y_{n} \rightarrow y$ and $y_{n}^{\prime} \rightarrow y^{\prime}$. Since $\alpha^{n}\left(k^{\prime \prime}\right)$ is eventually disjoint from any finite set of indices we have $d\left(y_{n}, y_{n}^{\prime}\right) \rightarrow 0$ so $y=y^{\prime}$. Since $\phi$ is continuous $\phi\left(y_{0}\right)=\phi\left(y_{n}\right) \rightarrow \phi(y)$ so $\phi\left(y_{0}\right)=\phi(y)$ and similarly $\phi\left(y_{0}^{\prime}\right)=\phi\left(y^{\prime}\right)$ so we conclude $\phi\left(y_{0}\right)=\phi\left(y_{0}^{\prime}\right)$. Thus $\phi$ does not depend on co-ordinates in $k^{\prime \prime}$, again contradicting essentiality. This establishes our claim that $x_{2} \in\left(X^{*}\right)^{k^{\prime}}$.

Lemma 3.5(a) now tells us that $\overline{\mathcal{O}}\left(\left(x_{1}, x_{2}\right), U \times U\right)$ is a union of POOD's on $X^{k \cup k^{\prime}}$ each of which has no links within $k$. Let $\Delta$ denote any one of these POOD's. The arguments we just used to show that $X_{2} \in\left(X^{*}\right)^{k^{\prime}}$ can be applied again to show that there is a (potentially) one-to-many map $\alpha$ from $k$ to $k^{\prime}$ such that $\Delta$ links co-ordinate $i \in k$ with all co-ordinates in $\alpha(i), \alpha(i) \cap \alpha(j) \neq \varnothing$ for $i \neq j$ and the range of $\alpha$ is all of $k^{\prime}$. We claim that in fact $\alpha(i)$ is a singleton for each $i \in k$. To see this let us call $i_{0}, \ldots, i_{r-1}$ a cycle if $i_{j+1} \in \alpha\left(i_{j}\right)$ for $j=0, \ldots, r-1$, with the convention that $i_{r}=i_{0}$. Now, the disjointness property of $\alpha$ implies the following fact: if $i_{0}, i_{1}, \ldots$ is an infinite sequence in $k$ with $i_{j+1} \in \alpha\left(i_{j}\right)$ then either the $i_{j}$ are pairwise distinct or $i_{r}=i_{0}$ for some $r$. In other words, beginning at $i_{0}$ cycling can occur only by coming back to $i_{0}$ itself. Moreover, different cycles are disjoint. Now suppose the cardinality of $\alpha\left(i_{0}\right)$ is two or more. If $i_{0}$ does not belong to a cycle then we immediately have that $i_{0}, \alpha\left(i_{0}\right), \ldots$ are distinct so our previous argument shows that $\phi$ does not depend on the co-ordinate $i_{0}$. If $i_{0}$ does belong to a cycle simply choose $i_{0}^{\prime} \in \alpha\left(i_{0}\right)$ so that $i_{0}^{\prime}$ does not belong to the cycle of $i_{0}$ and again we have $i_{0}^{\prime}, \alpha\left(i_{0}^{\prime}\right), \ldots$ distinct. Thus in either case we have contradicted the essentiality of $\phi$, establishing our claim that $\alpha$ is actually a $1-1$, onto map from $k$ to $k^{\prime}$. Thus there is an $\mathbf{m}: \mathbf{k} \rightarrow \mathbb{Z}$ such that $\Delta=\left\{(x, U(\alpha, \mathbf{m}) x): x \in X^{k}\right\}$.

In particular we may take $\Delta$ to be a POOD in $\overline{\mathcal{O}}\left(\left(x_{1}, x_{2}\right), U \times U\right)$ with $\left(x_{1}, x_{2}\right) \in \Delta$ and we conclude that $x_{2}=U(\alpha, \mathbf{m}) x_{1}$ so $x_{2} \in E$ also. Moreover $\Delta \subset R$ so $U(\alpha, \mathbf{m}) \in G$. 
Summarizing we have shown that $E$ is $R$-saturated and

$$
R \cap E \times E=R_{G} \cap E \times E .
$$

Next we want to show that $G$ is compact. For $U(\alpha, \mathbf{m}) \in G(k)$ we write $\pi(U(\alpha, \mathbf{m}))=$ $\alpha$ and $\mathbf{l}(U(\alpha, \mathbf{m}))=\mathbf{m}$. $\pi$ is a homomorphism from $G(k)$ to the permutation group $S(k)$. Setting $\tilde{G}=\pi(G)$ we claim that $\tilde{G}$ has finite orbits as a group of permutations of $k$. Indeed if $\tilde{G}(i)$ is infinite for some $i \in k$ there are $U\left(\alpha_{1}, \mathbf{m}_{1}\right), U\left(\alpha_{2}, \mathbf{m}_{2}\right), \ldots \in G$ such that $\alpha_{1}(i), \alpha_{2}(i), \ldots$ are distinct. If $x_{1}$ and $x_{2}$ in $X^{k}$ differ only at $i, U\left(\alpha_{n}, \mathbf{m}_{n}\right) x_{1}$ and $\left.U\left(\alpha_{n}, \mathbf{m}_{n}\right) x_{2}\right)$ differ only at $\alpha_{n}(i)$ so $d\left(U\left(\alpha_{n}, \mathbf{m}_{n}\right) x_{1}, U\left(\alpha_{n}, \mathbf{m}_{n}\right) x_{2}\right) \rightarrow 0$. Since $\phi\left(x_{i}\right)=\phi\left(U\left(\alpha_{n}, m_{n}\right) x_{i}\right), i=1,2$, we conclude that $\phi(x)=\phi\left(x^{\prime}\right)$, contradicting essentiality of $\phi$.

Next we claim that for $V \in G, i \in k$, if $\pi(V)(i)=i$ then $\mathbf{l}(V)(i)=0$. If this were not the case let $k_{1} \subset k$ be the union of the cycles of $\pi(V)$ over which $\mathrm{I}(V)$ has a non-zero sum, so $i \in k_{1}$. Write $V=V_{1} \times V_{2}$ on $X^{k_{1}} \times X^{k_{2}}, k_{2}=k-k_{1}$. Let $k_{1, i} \nearrow k_{1}$ and $k_{2, i} \nearrow k_{2}$ be finite unions of cycles of $\pi(V)$ and $N_{i}>0$ be such that $V^{N_{i}}$ has the form

$$
T^{\mathbf{m}_{1}} \times U_{1, i} \times \mathrm{id} \times U_{2, i} \quad \text { on } \quad X^{k_{1, l}} \times X^{k_{1}-k_{1, i}} \times X^{k_{2, i}} \times X^{k_{2}-k_{2, i}}
$$

for some $\mathbf{m}_{i}: k_{1, i} \rightarrow \mathbb{Z}-\{0\}$. Now if $\left(x_{1}, x_{2}\right) \in X^{k_{1}} \times X^{k_{2}}$ and $x_{1}$ has no pair of coordinates on the same $T$-orbit we have

$$
\overline{\mathcal{O}}\left(\left(x_{1}, x_{2}\right), V\right) \supset \overline{\mathcal{O}}\left(\left(x_{1}, x_{2}\right), T^{\mathrm{m}_{i}} \times U_{1, i} \times \mathrm{id} \times U_{2, i}\right)
$$

and denoting projection on $X^{k_{1, i}} \times X^{k_{2, i}}$ by $\sigma_{i}$ and the projection of $x_{2}$ on $X^{k_{2, i}}$ by $x_{2, i}$

$$
\sigma_{i} \overline{\mathcal{O}}\left(\left(x_{1}, x_{2}\right), V\right) \supset X^{k_{1, i}} \times\left\{x_{2, i}\right\}
$$

This implies that

$$
\overline{\mathcal{O}}\left(\left(x_{1}, x_{2}\right), V\right) \supset X^{k_{1}} \times\left\{x_{2}\right\} .
$$

Now $\phi\left(x_{1}, x_{2}\right)=\phi(y)$ for any $y \in \overline{\mathcal{O}}\left(\left(x_{1}, x_{2}\right), V\right)$. Thus $\phi\left(x_{1}, x_{2}\right)=\phi\left(x_{1}^{\prime}, x_{2}\right)$ for any $x_{1}^{\prime} \in X^{k_{1}}$ so $\phi$ does not depend on the co-ordinates in $k_{1}$. This conflict establishes our claim. As a consequence, if $V_{1}, V_{2} \in G$ and $\pi\left(V_{1}\right)(i)=\pi\left(V_{2}\right)(i)$ then $\mathbf{l}\left(V_{1}\right)(i)=$ $\mathrm{l}\left(V_{2}\right)(i)$, because $\pi\left(V_{2}^{-1} V_{1}\right)(i)=i$ so

$$
0=\mathbf{l}\left(V_{2}^{-1} V_{1}\right)(i)=-\mathbf{I}\left(V_{2}\right)(i)+\mathbf{l}\left(V_{1}\right)(i)
$$

We can now complete the proof that $G$ is compact. If $V_{1}, V_{2}, \ldots \in G$ then, since $\tilde{G}$ has finite orbits, we can find a subsequence $n_{j}$ such that for each $i \in k, \pi\left(V_{n_{j}}\right)(i)$ is constant for sufficiently large $j$. Define this constant to be $\alpha(i)$. It follows that $\mathbf{l}\left(V_{n_{j}}\right)(i)$ is also a constant, say $\mathbf{m}(i)$, for large $j$ and $V_{n_{j}} \rightarrow U(\alpha, \mathbf{m})$ uniformly. This establishes the compactness of $G$. If $V \in G$ then

$$
\phi U V U^{-1}=S \phi V U^{-1}=S \phi U^{-1}=\phi U U^{-1}=\phi
$$

so $U V U^{-1} \in G$. Thus $U G U^{-1} \subset G$ and similarly $U^{-1} G U \subset G$ so $U G U^{-1}=G$.

Finally, let $\sigma: X^{k} \rightarrow X^{k} / G$ denote the projection. Since $R \supset R_{G}, \phi$ factors

$$
\phi: X^{k} \stackrel{\sigma}{\longrightarrow} X^{k} / G \stackrel{\theta}{\longrightarrow} Y .
$$

As $E$ is $G$-invariant and we have shown $R \cap E \times E=R_{G} \cap E \times E$ and $E$ is $R$ saturated, $\theta^{-1} \theta \sigma(x)=\sigma(x)$ for $x \in E$. Thus to complete the proof of the theorem it 
suffices to show that $\sigma(E)$ is a dense $G_{\delta}$ in $X / G$. Now

$$
E^{c}=\left(\left(X^{*}\right)^{k}\right)^{c} \cup \bigcup_{\beta} \Delta_{\beta}
$$

where the $\Delta_{\beta}$ are the countably many POOD's which link precisely two co-ordinates.

We claim $G\left(\Delta_{\beta}\right)$ is a closed nowhere dense set. Indeed suppose $\Delta_{\beta}=$ $\left\{x \in X^{k}: x(j)=T^{t} x(i)\right\}$. Then for $U(\alpha, \mathbf{m}) \in G$

$$
U(\alpha, \mathbf{m}) \Delta_{\beta}=\left\{x \in X^{k}: x(\alpha(j))=T^{t+\mathbf{m}(j)-\mathbf{m}(i)} x(\alpha(i))\right\} .
$$

Since $\tilde{G}$ has finite orbits there are only finitely many possibilities for $\alpha(j)$ and $\alpha(i)$ and moreover $\alpha(j)$ and $\alpha(i)$ determine $\mathbf{m}(j)$ and $\mathbf{m}(i)$. Thus we see that $G \Delta_{\beta}$ is actually a finite union of sets $U(\alpha, \mathbf{m}) \Delta_{\beta}$, so it is closed and nowhere dense.

Now suppose that $\left(X^{*}\right)^{c}=\bigcup_{n} F_{n}, F_{n}$ closed nowhere dense sets. Then

$$
\left(\left(X^{*}\right)^{k}\right)^{c}=\bigcup_{i \in k} \bigcup_{n}\left(X^{*}\right)^{k-\{i\}} \times F_{n} .
$$

Now $G\left(\left(X^{*}\right)^{k-\{i\}} \times F_{n}\right)$ is closed and nowhere dense by the argument we just gave - it is actually a finite union of $G$-translates of $\left(X^{*}\right)^{k-\{i\}} \times F_{n}$.

Putting these facts together, $E^{c}=G\left(E^{c}\right)=\bigcup_{n} C_{n}$ where the $C_{n}$ are closed nowhere dense $G$-invariant sets. Then, since $E$ is $G$-invariant

$$
\sigma(E)^{c}=\sigma\left(E^{c}\right)=\bigcup_{n} \sigma\left(C_{n}\right)
$$

and since the $C_{n}$ are closed, nowhere dense and $G$-invariant, the $\sigma\left(C_{n}\right)$ are closed and nowhere dense in $X / G$. This completes the proof of the theorem.

We now state a couple of corollaries to the proof of theorem 3.6 which correspond to results in $[\mathbf{R u}]$.

Corollary 3.7. If $V, V^{\prime} \in G$ and $\pi(V)(i)=\pi\left(V^{\prime}\right)(i)$ for some $i$ then $\mathrm{I}(V)(i)=$ $\mathbf{l}\left(V^{\prime}\right)(i)$.

Corollary 3.8. $\tilde{G}=\pi(G)$ has finite orbits on $k$.

We equip $S(k)$, the group of permutations of $k$, with the topology of pointwise convergence.

Corollary 3.9. $\pi: G \rightarrow \tilde{G}$ is continuous.

Theorem 3.10 below corresponds to theorem 3.13 of [Ru]. It completes our development of the key points of a theory corresponding to that of $\S 3$ of [Ru]. As we have already stated the rest of the development in [Ru] proceeds along formal algebraic lines and can be carried out in precisely the same way in our setting.

THEOREM 3.10. Suppose $U=U(\pi, \mathrm{l})$ and $U^{\prime}=U\left(\pi^{\prime}, \mathrm{l}^{\prime}\right)$ on $X^{k}$ and $X^{k^{\prime}}$ are ergodic $\pi, \pi^{\prime}$ compact and $\phi:\left(X^{k}, U\right) \rightarrow(Y, S), \phi^{\prime}:\left(X^{k^{\prime}}, U^{\prime}\right) \rightarrow\left(Y^{\prime}, S^{\prime}\right)$ are essential homomorphisms. Set $G=G(\phi)$ and $G^{\prime}=G\left(\phi^{\prime}\right)$. Suppose that $(Y, S)$ and $\left(Y^{\prime}, S^{\prime}\right)$ are weakly isomorphic via a map $\psi$. Then there is a $U(\alpha, \mathbf{m}): X^{k} \rightarrow X^{k^{\prime}}$ such that the following diagram commutes (for all points for which the appropriate compositions are defined). 


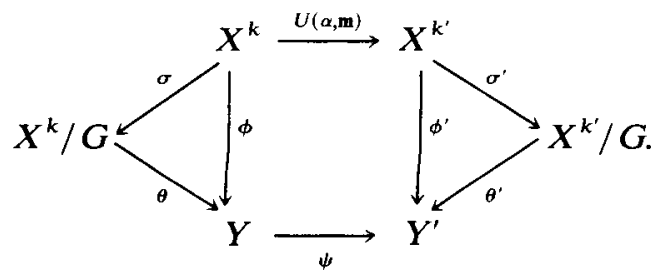

Moreover $U(\alpha, m)$ takes $G$-orbits to $G^{\prime}$-orbits and induces a (strict) isomorphism of $\left(X^{k} / G, U\right)$ and $\left(X^{k^{\prime}} / G^{\prime}, U^{\prime}\right)$ which lifts $\psi$.

Proof. Since $\theta$ and $\theta^{\prime}$ are almost 1-1 factor maps they are also weak isomorphisms so $\psi$ lifts to a weak isomorphism $\hat{\psi}: F \rightarrow F^{\prime}$ of $\left(X^{k} / G, U\right)$ and $\left(X^{k^{\prime}} / G^{\prime}, U^{\prime}\right)$, where $F$ and $F^{\prime}$ are invariant dense $G_{\delta}$ 's in $X^{k} / G$ and $X^{k^{\prime}} / G^{\prime}$.

As in the proof of theorem 3.6 let $E \subset X^{k}$ be the intersection of $\left(X^{*}\right)^{k}$ and those points having no two co-ordinates on the same $T$-orbit. Let $E^{\prime}$ be the corresponding set in $X^{k^{\prime}}$. As in the proof of theorem 3.6, $\sigma(E)$ and $\sigma^{\prime}\left(E^{\prime}\right)$ are dense $G_{\delta}$ 's in $X^{k} / G$ and $X^{k^{\prime}} / G^{\prime}$. It follows that we may choose $\sigma\left(x_{0}\right) \in \sigma(E)$ with $\hat{\psi} \sigma\left(x_{0}\right)=\sigma^{\prime}\left(x_{0}^{\prime}\right) \in$ $\sigma^{\prime}\left(E^{\prime}\right)$.

Now since $\hat{\psi} \sigma\left(x_{0}\right)=\sigma^{\prime}\left(x_{0}^{\prime}\right)$,

$$
\psi \phi x_{0}=\psi \theta \sigma x_{0}=\theta^{\prime} \hat{\psi} \sigma x_{0}=\theta^{\prime} \sigma^{\prime} x_{0}^{\prime}=\phi^{\prime} x_{0}^{\prime} .
$$

It follows that

$$
\sigma^{-1}(F) \times\left(\sigma^{\prime}\right)^{-1} F^{\prime} \cap \overline{\mathcal{O}}\left(\left(x_{0}, x_{0}^{\prime}\right), U \times U^{\prime}\right) \subset\left\{\left(x, x^{\prime}\right): \psi \phi x=\phi^{\prime} x^{\prime}\right\} .
$$

Note that $\sigma^{-1}(F)$ and $\left(\sigma^{\prime}\right)^{-1} F^{\prime}$ are dense $G_{\delta}$ 's by compactness of $G$ and $G^{\prime}$. By lemma $3.5, \bar{O}\left(\left(x_{0}, x_{0}^{\prime}\right), U \times U^{\prime}\right)$ contains a POOD $\Delta$ which has no links within $k$ or $k^{\prime}$. We claim that $\Delta$ is actually the graph of a $U(\alpha, \mathbf{m}): X^{k} \rightarrow X^{k^{\prime}}$.

To see this observe that $\Delta$ has the following form: there are $k_{1} \subset k, k_{1}^{\prime} \subset k^{\prime}$ and $U(\alpha, \mathbf{m}): X^{k_{1}} \rightarrow X^{k_{1}^{\prime}}$ such that

$$
\Delta=\left\{\left(x, x^{\prime}\right): U(\alpha, \mathbf{m}) \tau_{1} x=\tau_{1}^{\prime} x^{\prime}\right\},
$$

$\tau_{1}$ and $\tau_{1}^{\prime}$ the projections on $X^{k_{1}}$ and $X^{k_{1}^{\prime}}$. What we are trying to show is that $k_{1}=k$ and $k_{1}^{\prime}=k^{\prime}$. By [O, theorem 15.1], $\tau_{1}\left(\sigma^{-1}(F)\right)$ contains a dense $G_{\delta}$ in $X^{k_{1}}$ so we see that

$$
W=\left\{x \in \sigma^{-1}(F): \exists x^{\prime} \in\left(\sigma^{\prime}\right)^{-1} F^{\prime},\left(x, x^{\prime}\right) \in \Delta\right\}
$$

contains a dense $G_{\delta}$ in $X^{k}$. Suppose that $k_{1} \neq k$. Now, if $x, \bar{x} \in W$ and $x(j)=\bar{x}(j)$ for $j \in k_{1}$ then, choosing $x^{\prime} \in\left(\sigma^{\prime}\right)^{-1} F^{\prime}$ with $\left(x, x^{\prime}\right) \in \Delta$, we have also $\left(\bar{x}, x^{\prime}\right) \in \Delta$. Since $x, \bar{x} \in \sigma^{-1} F$ and $x^{\prime} \in\left(\sigma^{\prime}\right)^{-1} F^{\prime}$ we conclude that $\psi \phi x=\phi^{\prime} x^{\prime}=\psi \phi \bar{x}$ whence $\phi x=\phi \bar{x}$. For $z \in X^{k_{1}}$ set $W_{z}=W \cap \tau_{1}^{-1}\{z\}$. We have shown that $\phi$ is a constant on sets $W_{z}$. When $W_{z}$ is dense in $\{z\} \times X^{k-k_{1}} \phi$ is constant on $\{z\} \times X^{k-k_{1}}$ by continuity. Since this occurs for a dense set of $z$ by [O, theorem 15.1], we conclude that $\phi$ is constant on $X^{k-k_{1}} \times\{z\}$ for all $z \in X^{k_{1}}$. That is, $\phi$ does not depend on co-ordinates in $k-k_{1}$, contradicting essentiality. It follows that $k_{1}=k$, and similarly $k_{1}^{\prime}=k^{\prime}$.

Thus $\Delta$ is the graph of $U(\alpha, \mathbf{m}): X^{k} \rightarrow X^{k^{\prime}}$. It follows that $\psi \phi x=\phi^{\prime} U(\alpha, \mathbf{m}) x$ for $x$ in the dense $G_{\delta} W=\left\{x \in \sigma^{-1}(F): U(\alpha, \mathbf{m}) x \in\left(\sigma^{\prime}\right)^{-1} F^{\prime}\right\}$. Thus $U(\alpha, \mathbf{m})$ provides the desired lifting of $\psi$. 
Writing $U(\alpha, \mathbf{m})=\tilde{U}$, we have shown that $\sigma^{\prime} \tilde{U} x=\hat{\psi} \sigma x$ for $x$ in $W$. In particular $\tilde{U} \times \tilde{U}\left(R_{G} \cap W \times W\right) \subset R_{G^{\prime}}$. Now if $x, y \in X^{k}$ with $y=g x, g \in G$, since $W \cap g^{-1} W$ is a dense $G_{\delta}$ we may choose $x_{n} \in W \cap g^{-1} W$ so that $x_{n} \rightarrow x$. Since $\left(x_{n}, g x_{n}\right) \in W \times W$ we have $\left(\tilde{U} x_{n}, \tilde{U} g x_{n}\right) \in R_{G^{\prime}}$. But $\left(\tilde{U} x_{n}, \tilde{U} g x_{n}\right) \rightarrow(\tilde{U} x, \tilde{U} y)$ so $(\tilde{U} x, \tilde{U} y) \in R_{G^{\prime}}$. Thus $(\tilde{U} \times \tilde{U}) R_{G} \subset R_{G^{\prime}}$ and by symmetry $(\tilde{U} \times \tilde{U}) R_{G}=R_{G^{\prime}}$, that is $\tilde{U}$ takes $G$-orbits to $G^{\prime}$-orbits. Thus $\tilde{U}$ induces a homeomorphism of $X^{k} / G$ and $X^{k^{\prime}} / G^{\prime}$ which agrees with $\hat{\psi}$. Since $\hat{\psi}$ is equivariant on its domain $F$ which is dense, we conclude that $\tilde{U}$ is equivariant everywhere.

We conclude this section with some remarks on the connection between AMPJ and Auslander and Markley's notion of a graphic flow $[\mathbf{M}],[\mathbf{A M}] .(X, T)$ is called graphic if it is minimal and the only minimal subsets of $(X \times X, T \times T)$ are offdiagonals. The following proposition is due to Markley.

Proposition 3.11. If $(X, T)$ is minimal and has AMSJ then it is graphic.

Proof. Let $M$ be a minimal subset of $X \times X$ and $\pi_{1}: M \rightarrow X$ the projection on the first co-ordinate. ( $\pi_{1}$ is onto by minimality of $\left.X\right) \cdot \pi_{1}^{-1}\left(X^{*}\right)$ is a non-empty invariant set in $M$, hence it is dense in $M$ by minimality. Thus $\pi_{1}^{-1}\left(X^{*}\right)$ is a dense $G_{\delta}$ in $M$. Similarly $\pi_{2}^{-1}\left(X^{*}\right)$ is a dense $G_{\delta}$ in $M$ where $\pi_{2}$ denotes the projection on the second co-ordinate. Thus $\pi_{1}^{-1}\left(X^{*}\right) \cap \pi_{2}^{-1}\left(X^{*}\right)$ is non-empty so there is an $\left(x_{1}, x_{2}\right) \in$ $M \cap X^{*} \cap X^{*}$. Thus $M=\overline{\mathcal{O}}\left(\left(x_{1}, x_{2}\right), T \times T\right)$ is an off-diagonal (it is not $X \times X$ because $X \times X$ is not minimal).

It is worth noting that AMPJ does not imply minimality. Indeed one can mimic the construction of a rank one mixing map as carried out in [Ru] to obtain a subshift of $\{0,1\}^{\mathbf{Z}}$ which has a fixed point (corresponding to the 'spacer' symbol) but has AMPJ. This was a suggestion of Ken Berg.

Finally graphic does not imply SAMPJ, as has been observed by Auslander. To see this take any proximal but not almost 1-1 extension of a graphic flow $(X, T)$. The resulting flow is graphic by a result of $[\mathbf{A}, \mathbf{M}]$ but does not have SAMPJ by proposition 3.2 .

\section{Real flows}

If $\left(X,\left\{T_{t}\right\}\right)$ is an $\mathbb{R}$-flow, $k$ is a finite or countable set and $\mathbf{a}: k \rightarrow \mathbb{R}$ we write $T^{\mathrm{a}}=\bigotimes_{i \in k} T_{a(i)}$. By an off-diagonal for $\left\{T_{t}\right\}$ we mean any set $T^{\mathrm{a}} \Delta, \Delta$ the diagonal in $X^{k}$ and a POOD has the obvious meaning. We say $\left(X,\left\{T_{t}\right\}\right)$ has almost minimal self-joinings (AMSJ) if there is an invariant dense $G_{\delta} X^{*}$ in $X$ such that for all finite $k$ and $x \in\left(X^{*}\right)^{k}, \bar{O}\left(x,\left\{T^{1 t}\right\}\right)$ is a POOD. Setting $X^{\prime}=X-X^{*}$ we say $\left(X,\left\{T_{t}\right\}\right)$ has strongly almost minimal self-joining (SAMSJ) if for all finite $k$ and $k^{\prime}$ and $x \in\left(X^{*}\right)^{k}, x^{\prime} \in\left(X^{\prime}\right)^{k^{\prime}}$

$$
\overline{\mathcal{O}}_{+\infty}\left(\left(x, x^{\prime}\right), T^{\mathbf{1}}\right)=\overline{\mathcal{O}}_{+\infty}\left(x, T^{\mathbf{l}}\right) \times\left(x^{\prime}, T^{\mathbf{l}}\right) .
$$

Proposition 4.1. Let $\left\{T_{t}\right\}$ be defined as the flow over Chacón's example under the function $\alpha 1_{\{x: x(0)=0\}}+1_{\{x: x(0)=1\}}$ where $\alpha$ is irrational. Then $\left\{T_{t}\right\}$ is weak-mixing, POD and has SAMSJ. $X^{*}$ consists of all points whose orbits never pass through the bad set in the cross-section. 
We shall not prove this but mention only that it can be proved using topological versions of the ideas in $[\mathbf{J}, \mathbf{P}]$.

We say $\left(X,\left\{T_{i}\right\}\right)$ has almost minimal re-scaling joinings (AMRJ) if, for all finite $k$, a : $k \rightarrow \mathbb{R}-\{0\}$, and $x \in\left(X^{*}\right)^{k}, \overline{\mathcal{O}}\left(x,\left\{T^{\mathbf{a} t}\right\}\right)$ is a POOD. Strongly almost minimal re-scaling joinings (SAMRJ) can be defined in an obvious way. The flow of 4.1 surely has SAMRJ but we have not been able to find a proof of this. It is not difficult to show, as in $[\mathbf{J}, \mathbf{P}]$, that distinct rescalings $T_{a_{1} t}$ and $T_{a_{2} t}$ are not isomorphic. In ergodic theory the general machinery of $[\mathbf{J}, \mathbf{R}]$ shows that minimal self-joinings and pairwise non-isomorphism of rescalings imply minimal rescaling joinings. We do not know if the analogous result in topological dynamics is valid. One way to show the example of 4.1 has SAMRJ would be to establish an obvious analogue of the result in $[\mathbf{J}, \mathbf{K}]$ and then use it together with (measure-theoretic) minimal re-scaling joinings as we did for the discrete-time Chacón flow in $\S 2$.

We will say $\left(X,\left\{T_{t}\right\}\right)$ is almost transitive if it has a single orbit which is a dense $G_{\delta}$.

Proposition 4.2. If $\left(X,\left\{T_{t}\right\}\right)$ has AMSJ then $\left(X,\left\{T_{t}\right\}\right)$ is either almost transitive or weak-mixing.

Proof. Suppose that $\left(X,\left\{T_{t}\right\}\right)$ is not almost transitive. Let $\Delta_{t}$ denote the off-diagonal $\left(T_{t} \times\right.$ id) $\Delta, \Delta$ the diagonal in $X^{2}$. We claim that $\bigcup_{t \in \mathbb{R}} \Delta_{t}$ is an $F_{\sigma}$ with empty interior. Since

$$
\bigcup_{i \in \mathbb{R}} \Delta_{t}=\bigcup_{i \in Z}\left(T_{i} \times \mathrm{id}\right)\left(\bigcup_{t \in[0,1]} \Delta_{t}\right),
$$

it suffices to show that the closed set $\bigcup_{t \in[0,1]} \Delta_{T}$ is nowhere dense. Suppose this were not the case, then there exist open sets $U$ and $V$ in $X$ such that $\bigcup_{t \in[0,1]}\left\{T_{t} x\right\} \supset U$ for $x \in V$. Since any $x^{\prime} \in X^{*}$ has dense orbit, the orbit of $x^{\prime}$ and $x$ are the same, that is $X^{*}$ is precisely the orbit of $x$ which means that $\left(X,\left\{T_{t}\right\}\right)$ is almost transitive, a contradiction.

It follows that $X^{*} \times X^{*}-\bigcup_{t \in \mathbf{R}} \Delta_{t}$ is a dense $G_{\delta}$ in $X \times X$ so we may choose $\left(x_{1}, x_{2}\right) \in X^{*} \times X^{*}$ with $x_{1}, x_{2}$ on different $\left\{T_{t}\right\}$-orbits. Thus $\tilde{\mathscr{O}}\left(\left(x_{1}, x_{2}\right),\left\{T_{t} \times T_{t}\right\}\right)$ is not an off-diagonal so by AMSJ it must be $X \times X$, establishing weak-mixing of $\left\{T_{1}\right\}$.

Proposition 4.3. If $\left(X, T_{t}\right)$ is weak-mixing with AMSJ then for $x \in\left(X^{*}\right)^{k}$ the discrete orbit closure $\overline{\mathcal{O}}\left(x, T^{\mathbf{1}}\right)$ is a POOD.

Proof. It suffices to assume that $x$ has no two co-ordinates on the same $\left\{T_{t}\right\}$-orbit and show that $\overline{\mathcal{O}}\left(x, T^{1}\right)=X^{k}$. In this case by AMSJ of $\left(X,\left\{T_{t}\right\}\right), \overline{\mathcal{O}}\left(x,\left\{T^{1 t}\right\}\right)=X^{k}$. Set $E=\bar{O}\left(x, T^{1}\right)$ and for each $n$ set $E_{n}=\bigcup_{0 \leq t \leq 1 / n} T^{1 t} E_{n}$. Since the closed set $\bigcup_{i=0}^{n-1} T^{1 i / n} E_{n}$ contains $\overline{\mathcal{O}}\left(x,\left\{T^{1 t}\right\}\right)$ it must be $X^{k}$. Thus some $T^{1 i / n} E_{n}$, and hence $E_{n}$ itself, has non-empty interior. Since $E_{n}$ is $T^{1}$-invariant and $T^{1}$ is ergodic (by weak-mixing of $\left.\left\{T_{t}\right\}\right) E_{n}=X^{k}$. It follows that $E=\bigcap_{n} E_{n}=X^{k}$.

Using proposition 4.3 one can establish results for AMSJ $\mathbb{R}$-flows similar to those of $\$ 3$ for maps. Having only AMSJ and not AMRJ imposes some unnatural restrictions so we simply restrict ourselves to stating some results for $T^{1}$ on $X^{k}, k$ finite. 
Proposition 4.4. Suppose $\left(X,\left\{T_{t}\right\}\right)$ is weak-mixing with AMSJ. Then the automorphism group of $\left(X^{k}, T^{1}\right)$ consists of the maps $S_{\pi} T^{2}$ where $\pi: k \rightarrow k$ is a permutation. In particular the automorphism group of $\left(X, T_{1}\right)$ is $\left\{T_{t}: t \in \mathbb{R}\right\}$. Suppose $\left(X,\left\{T_{t}\right\}\right)$ has SAMSJ. If $\phi:\left(X^{k}, T^{1}\right) \rightarrow(Y, S)$ is a factor map then there is a compact subgroup $G$ of the $S_{\pi} T^{\mathrm{a}}$, necessarily having finite orbits, such that $\phi$ is the composition of the projection $X^{k} \rightarrow X^{k} / G$ and an almost 1-1 factor map $X^{k} / G \rightarrow Y$. In particular $\left(X, T_{1}\right)$ has only almost 1-1 factors.

For both $\mathbb{Z}$ and $\mathbb{R}$-flows the question remains whether we can obtain minimal self-joinings with no exceptional set. For $\mathbb{R}$-flows certain horocycle flows supply examples, as can be shown using results of Ratner [Ra]. Say $\left(X,\left\{T_{t}\right\}\right)$ has $k$-fold minimal self-joinings (MSJ) if for all $x \in X^{k}, \overline{\mathcal{O}}\left(x, T^{1 t}\right)$ is a POOD.

THEOREM 4.5. If $\left(X, T_{t}\right)$ is a horocycle flow defined by a maximal discrete co-compact non-arithmetic subgroup of $\mathrm{SL}_{2}(\mathbb{R})$ then $\left\{h_{t}\right\}$ has 2-fold MSJ.

Proof. It suffices to show that for $x, y$ on different orbits $E=\bar{O}\left((x, y),\left\{T_{t} \times T_{t}\right\}\right)=X^{2}$. Let $\lambda$ be an invariant ergodic measure supported on $E$. By unique ergodicity of $\left\{T_{t}\right\}$ both projections of $\lambda$ are the unique invariant measure $\mu$ for $\left\{T_{t}\right\}$. Since $\left\{T_{t}\right\}$ has measure-theoretic MSJ ([Ra]) we conclude that $\lambda$ is either $\mu \times \mu$ or an off-diagonal measure $\left(I \times T_{t_{0}}\right) \mu_{\Delta}, \mu_{\Delta}$ the diagonal measure on $X \times X$. If $\lambda=\mu \times \mu$, supp $\lambda=$ $X \times X \subset E$ so we are done.

Suppose then that $\lambda=\left(I \times T_{t_{0}}\right) \mu_{\Delta}$ so

$$
\operatorname{supp} \lambda=\left\{\left(z, T_{t_{0}} z\right): z \in X\right\}=\Delta_{t_{0}} \subset E \text {. }
$$

This means that for all $\delta>0$ there is a $t$ with $d\left(T^{t} x, T^{t-t_{0}} y\right)<\delta$. Fix $p \in \mathbb{Z}^{+}$. For $\varepsilon>0$, by the $H_{p}$ property of $\left\{T_{t}\right\}$ ([Ra]), if $\delta$ is sufficiently small there is an such that either

$$
d\left(T^{t+s} x, T^{t+s-t_{0}+p} y\right)<\varepsilon
$$

or

$$
d\left(T^{t+s} x, T^{t+s-t_{0}-p} y\right)<\varepsilon .
$$

As $\varepsilon \rightarrow 0$ either $p$ or $-p$ will occur infinitely often so we conclude that for each $p \in \mathbb{Z}^{+}, E$ contains either the off-diagonal $\Delta_{t_{0}+p}$ or $\Delta_{t_{0}-p}$. Thus there are sets $A^{+}$and $A^{-}$in $\mathbb{Z}^{+}$such that $A^{+} \cup A^{-}=\mathbb{Z}^{+}$and for each $z \in X$

$$
\left(\{z\} \times X \cap E \supset\{z\} \times\left(\left\{T_{t_{0}+p} z: p \in A^{+}\right\} \cup\left\{T_{t_{0}-p} z: p \in A^{-}\right\}\right) .\right.
$$

Suppose now that $A^{+}$contains arbitrarily long intervals. By minimality of $T_{1}$ we conclude that

$$
(\{z\} \times X) \cap E=\{z\} \times X
$$

for all $z \in X$ so $E=X \times X$. If $A^{+}$does not contain arbitrarily long intervals then $A^{-}$has gaps of length less than some $l$. By minimality of $T_{-1}$ it follows that

$$
\bigcup_{i=0}^{l} h_{-i}\left\{T_{t_{0}-p} z: p \in A^{-}\right\}^{-}=X
$$

so $\left\{T_{t_{0}-p} z: p \in A^{-}\right\}^{-}$has interior for all $z$. Choose a countable base $\left\{U_{i}\right\}$ for the topology of $X$ and for each $z$ choose a $U_{i(z)}$ such that $U_{i(z)} \subset\left\{T^{t_{0}-p} z: p \in A^{-}\right\}^{-}$. Let 
$X_{i}=\{z: i(z)=i\}$ so $X=\bigcup_{i} X_{i}$ whence the closure $\vec{X}_{i}$ of some $X_{i}$ has non-empty interior. If $\bar{X}_{i}$ contains a non-empty open set $V$ then since $E \supset X_{i} \times U_{i}$

$$
E \supset\left(X_{i} \times U_{i}\right)^{-} \supset V \times U_{i} \text {. }
$$

Thus $E$ has non-empty interior and by ergodicity of $\left\{h_{t} \times h_{t}\right\}$ we conclude $E=X \times X$.

We conjecture that any horocycle flow as in theorem 4.5 actually has $k$-fold MSJ for all $k$. It does not have minimal re-scaling joinings, or even AMRJ, since $\left\{\boldsymbol{h}_{\boldsymbol{t}}\right\}$ and $\left\{h_{a t}\right\}$ are isomorphic for $a>0$.

A number of questions are left open by this paper. Do there exist $\mathbb{Z}$-flows with unrestricted (that is, no exceptional set) MSJ or MPJ? Does MSJ or AMSJ for $\mathbb{Z}$-flows together with non-isomorphism of $T$ and $T^{-1}$ imply MPJ or AMPJ, as it does in ergodic theory? Does 2-fold MSJ or AMSJ for $\mathbb{Z}$ - or R-flows imply 3-fold MSJ or AMSJ? Here the answer is not known in ergodic theory either. Is SAMPJ necessary for the results on factors or will AMPJ suffice?

\section{REFERENCES}

[A, M] J. Auslander \& N. Markley. Graphic minimal sets. Preprint.

$[\mathbf{F}, \mathbf{K}, \mathbf{S}] \quad$ H. Furstenberg, H. Keynes \& L. Shapiro. Prime flows in topological dynamics. Israel J. Math. 14 (1973), 26-38.

[J] A. del Junco. A family of counterexamples in ergodic theory. Israel J. Math. 44 (1983), 160-188.

[J, K] A. del Junco \& M. Keane. On generic points in the cartesian square of Chacón's transformation. To appear in Ergod. Th. \& Dynam. Sys.

[J, P] A. del Junco \& K. Park. An example of a measure-preserving flow with minimal self-joinings. J. d'Analyse Math. 42 (1983), 99-211.

[J, R] A. del Junco \& D. Rudolph. On ergodic actions whose self-joinings are graphs. Preprint.

$[\mathbf{J}, \mathbf{R}, \mathbf{S}] \quad$ A. del Junco, A. M. Rahe \& L. Swanson. Chacón's automorphism has minimal self-joinings. J. d'Analyse Math. 37 (1980), 276-284.

[M] N. Markley. Topological minimal self-joinings. Preprint.

[Ra] M. Ratner. Joinings of horocycle flows. Preprint.

[Ru] D. R. Rudolph. An example of a measure-preserving map with minimal self-joinings, and applications. J. d'Analyse Math. 35 (1979), 97-122. 